\title{
Phytoprotection
}

\section{Les pourridiés des arbres : un secret bien gardé}

\section{Gaston Laflamme}

Volume 86, numéro 1, avril 2005

URI : https://id.erudit.org/iderudit/011713ar

DOI : https://doi.org/10.7202/011713ar

Aller au sommaire du numéro

\section{Éditeur(s)}

Société de protection des plantes du Québec (SPPQ)

ISSN

0031-9511 (imprimé)

1710-1603 (numérique)

Découvrir la revue

\section{Citer cet article}

Laflamme, G. (2005). Les pourridiés des arbres : un secret bien gardé. Phytoprotection, 86(1), 37-42. https://doi.org/10.7202/011713ar

\section{Résumé de l'article}

Les pourridiés des arbres sont des maladies qui engendrent des caries de racines et/ou des attaques du cambium au niveau du collet. Bien que la fin soit catastrophique pour l'arbre, une grande partie du cycle de vie des champignons demeure invisible. Au Québec, les données de pertes ligneuses dues aux pourridiés sont fragmentaires. Un rapport du Service canadien des forêts estime que les décroissances par les maladies et les insectes des arbres au Québec sont respectivement de $36 \%$ et $64 \%$, sans aucune perte par les pourridiés. Par comparaison, en Ontario où les pourridiés ont été étudiés, le pourcentage de perte par les maladies se situe à $65 \%$, soit la situation inverse par rapport au Québec. Et même en Ontario, les pertes occasionnées par les pourridiés seraient sous-estimées car souvent attribuées à d'autres causes comme des insectes défoliateurs ou les chablis. Les interventions en forêt augmentent parfois l'impact de ces agents pathogènes. Ainsi, le dégagement de la régénération de conifères a favorisé les attaques par des champignons sur les arbres résiduels avec un résultat contraire à celui escompté. Aussi, la coupe des arbres crée une niche écologique qui n'existe pas naturellement en forêt : les souches. Celles-ci sont colonisées par un grand nombre de champignons dont certains sont pathogènes; ainsi, les éclaircies dans les pinèdes rouges favorisent la colonisation par Heterobasidion annosum si les souches ne sont pas traitées. Les épinettes peuvent subir l'attaque d'Inonotus tomentosus si ces arbres sont en état de stress à cause de la densité du peuplement ou de la qualité du site. Enfin, la présence de l'armillaire commun sur des souches de feuillus peut anéantir les conifères plantés en périphérie.
Tous droits réservés @ La société de protection des plantes du Québec, 2005
Ce document est protégé par la loi sur le droit d'auteur. L’utilisation des services d’Érudit (y compris la reproduction) est assujettie à sa politique d'utilisation que vous pouvez consulter en ligne.

https://apropos.erudit.org/fr/usagers/politique-dutilisation/ 


\title{
Société de protection des plantes du Québec 97 ${ }^{\text {e }}$ Assemblée annuelle (2005) Quebec Society for the Protection of Plants 97 ${ }^{\text {th }}$ Annual meeting (2005)
}

Gatineau (Québec), 9 et 10 juin 2005 / Gatineau (Quebec), 9 and 10 June 2005

Symposium / Symposium

Santé des racines, santé des plantes

Healthy roots, healthy plants

\section{Les pourridiés des arbres : un secret bien gardé}

\author{
Gaston Laflamme ${ }^{1}$ \\ PHYTOPROTECTION 86 : 37-42
}

\begin{abstract}
Les pourridiés des arbres sont des maladies qui engendrent des caries de racines et/ou des attaques du cambium au niveau du collet. Bien que la fin soit catastrophique pour l'arbre, une grande partie du cycle de vie des champignons demeure invisible. Au Québec, les données de pertes ligneuses dues aux pourridiés sont fragmentaires. Un rapport du Service canadien des forêts estime que les décroissances par les maladies et les insectes des arbres au Québec sont respectivement de 36 et $64 \%$, sans aucune perte par les pourridiés. Par comparaison, en Ontario où les pourridiés ont été étudiés, le pourcentage de perte par les maladies se situe à $65 \%$, soit la situation inverse par rapport au Québec. Et même en Ontario, les pertes occasionnées par les pourridiés seraient sous-estimées car souvent attribuées à d'autres causes comme des insectes défoliateurs ou les chablis. Les interventions en forêt augmentent parfois l'impact de ces agents pathogènes. Ainsi, le dégagement de la régénération de conifères a favorisé les attaques par des champignons sur les arbres résiduels avec un résultat contraire à celui escompté. Aussi, la coupe des arbres crée une niche écologique qui n'existe pas naturellement en forêt: les souches. Celles-ci sont colonisées par un grand nombre de champignons dont certains sont pathogènes; ainsi, les éclaircies dans les pinèdes rouges favorisent la colonisation par Heterobasidion annosum si les souches ne sont pas traitées. Les épinettes peuvent subir l'attaque d'Inonotus tomentosus si ces arbres sont en état de stress à cause de la densité du peuplement ou de la qualité du site. Enfin, la présence de l'armillaire commun sur des souches de feuillus peut anéantir les conifères plantés en périphérie.
\end{abstract}

Mots clés : Carie rouge alvéolaire, Heterobasidion annosum, Inonotus tomentosus, Phlebiopsis gigantea, pourridié-agaric.

\section{[Root and butt rots of trees: a well-kept secret]}

Root and butt rots are hidden tree diseases. During a long part of their life cycle, these pathogens are mostly invisible in spite of their catastrophic final impact on trees. In Quebec, data on wood volume loss caused by root and butt rots are sketchy. A report from the Canadian Forest Service divided forest loss caused by diseases from that caused by insects in Quebec to respectively 36 and $64 \%$, without mentioning root and butt rots. In comparison with data from Ontario where root and butt rots have been studied, the percentage of losses caused by diseases is around $65 \%$, which is the opposite of the situation reported in Quebec. Moreover, losses through root and butt rots in Ontario could be underestimated as they are often reported under defoliators or windthrows. Forest treatments may increase the impact of root rot pathogens. For example, pre-commercial thinning in conifer stands has promoted the development of root rot pathogens on residual trees with a result opposite to the one expected. By cutting these trees, we are creating a new ecological niche that does not occur naturally, i.e., stumps. These

\footnotetext{
1. Ressources naturelles Canada, Service canadien des forêts, Sainte-Foy (Québec), Canada G1V 4C7; courriel : laflamme@rncan.gc.ca
} 
stumps are colonized by several fungi including pathogens. For example, thinning of red pine stands favours stump colonization by Heterobasidion annosum if these stumps are not treated. Spruces can become infected by Inonotus tomentosus if these trees are under stress, e.g., in a stand of high stem density or because they are planted on a low quality site. Finally, Armillaria root rot on stumps of hardwood may kill conifer seedlings planted nearby.

Keywords: Armillaria root rot, Heterobasidion annosum, Inonotus tomentosus, Phlebiopsis gigantea, red butt rot.

\section{INTRODUCTION}

Le terme "pourridié " est le nom générique de maladies qui engendrent la pourriture des racines et du collet et provoquent parfois la mort des arbres (Abgrall et Soutrenon 1991). On le traduit en anglais sous le vocable "root and butt rots". L'expression "carie de racine" (root decay) est aussi utilisée par opposition à "carie du tronc" (stem decay ou trunk rot). Ces expressions "carie de racine" et "carie du tronc" sont surtout en usage au Canada, provenant de la traduction du mot decay de textes anglais. II existe des maladies comme la carie du blé, causée par des champignons dits de charbon (Ustilaginales), mais qui n'ont rien à voir avec le processus de dégradation du bois. La décomposition du bois par les microorganismes est progressive; les expressions "coloration du bois" (discoloration), "carie au stade initial " (incipient decay) et "carie au stade avancé " (advanced decay), ou "final", décrivent ces différentes étapes. Des informations plus détaillées sur la dégradation du bois et des champignons responsables sont présentées dans Boulet (2003).

II est bien connu que les pourridiés peuvent causer la mort des arbres. Cependant, ils provoquent aussi une diminution de croissance des arbres qui n'est pas perceptible à moins de sonder les arbres à la tarière de Pressler. II faut donc comptabiliser dans la colonne des pourridiés ces pertes comme celles causées par les chablis d'arbres fortement affectés par des pourridiés.

\section{Études des caries des arbres}

Les trois principaux objectifs des études de caries des arbres vivants menées au Canada étaient de calculer les volumes de carie et de coloration du bois, d'identifier les champignons responsables de cette dégradation, et enfin d'appliquer des facteurs de rebut (cull). Ce facteur est appliqué au volume marchand récolté afin d'obtenir le volume réel utilisable, soit par I'industrie du sciage, soit par les usines de pâtes et de papiers. Pour certaines études, des observations sur les portes d'entrée possibles des microorganismes responsables des caries et des colorations des arbres ont aussi été rapportées (Laflamme et Lortie 1972; Lortie 1968).

L'étude de la carie et de la coloration se fait par la dissection des arbres: c'est donc une méthode destructive et laborieuse. Les arbres sont abattus et sectionnés afin de mesurer l'arbre sur toute sa longueur pour en calculer le volume. De plus, la dissection permet d'identifier les caries de racines et celles du tronc, de localiser les portes d'entrée de ces caries, de mesurer et d'échantillonner les portions montrant des colorations et caries du bois et d'en isoler les microorganismes responsables. L'étude la plus détaillée au Canada a eu lieu dans la province de I'Ontario; elle s'est déroulée sur une période allant de 1947 à 1957. Près de 23000 arbres de 18 essences différentes ont été disséqués et mesurés. Les résultats ont été publiés dans de nombreux rapports. Basham (1991) a résumé l'essentiel des informations tout en donnant les références pertinentes. Au Québec, l'étude la plus importante a été initiée par le Dr René Pomerleau. L'échantillonnage a eu lieu durant les périodes de 1939-1944 et 1954-57. Près de 6000 épinettes noires (Picea mariana (Mill.) B.S.P.) et 8400 sapins baumiers (Abies balsamea (L.) Mill.) ont été disséqués, mesurés et échantillonnés comme dans l'étude ontarienne. La compilation des résultats a été publiée séparément pour le sapin (Lavallée 1986) et l'épinette noire (Lavallée 1987). Dans la province de Terre-Neuve-Labrador, un relevé extensif sur les caries et colorations du bois a débuté au début des années 70, en même temps que l'inventaire forestier de cette province (Laflamme et al. 1977).

La tâche la plus difficile est d'isoler et d'identifier les champignons par des caractères morphologiques et culturaux, surtout que le nombre et la variété des espèces sont parfois assez impressionnants. Par exemple, chez les trembles (Populus tremuloides Michx. et $P$. grandidentata Michx.), plus de 260 espèces de champignons basidiomycètes associés aux caries et colorations du bois ont été identifiées en Amérique du Nord (Lindsey et Gilbertson 1978). L'identification des champignons de carie résultant de ces études nous permet d'apprécier l'incidence des pourridiés parmi les essences étudiées, même si ces études a priori ne visaient pas spécifiquement les caries de racines.

Des études sur les pourridiés des arbres ont été menées en Ontario par R.D. Whitney et M. Dumas, principalement, et en Colombie Britannique par W.J. Bloomberg, D.J. Morrison et leurs collaborateurs. Au Québec, mis à part une récente étude sur la maladie du rond, les observations n'ont été que ponctuelles, comme celles de Ouellette (1967) et Ouellette et Bard (1961), respectivement sur la carie rouge alvéolaire de l'épinette blanche (Picea glauca (Moench) Voss et sur le pourridié-agaric du pin gris (Pinus banksiana Lamb.).

\section{Importance relative des pertes ligneuses en forêt}

Dans I'Est canadien, on attribue principalement les pertes ligneuses aux insectes défoliateurs, surtout à la suite d'épidémies de tordeuse des bourgeons de l'épinette. Mais dans quelle proportion les autres insectes et champignons pathogènes contribuent à ces pertes? On trouve une certaine réponse à cette 
Tableau 1. Importance des pertes annuelles ligneuses en forêt par catégorie au Canada (millions de mètres cubes) (Hall et Moody 1994)

\begin{tabular}{lllll}
\hline Maladies & Insectes & Feux & Coupes & Total \\
\hline 51,2 & 51,6 & 36,0 & 160,0 & 298,8 \\
\hline
\end{tabular}

question dans l'un des rares rapports où l'on tente de colliger ces informations sur les pertes par les ravageurs forestiers au Canada (Hall et Moody 1994). Cette compilation couvre la période de 1982 à 1987. De façon tout à fait pertinente, les auteurs ajoutent aux pertes résultant de la mortalité, celles de la réduction de croissance due aux ravageurs.

Ainsi, au Canada, les pertes ligneuses causées par les maladies ou les insectes sont équivalentes pour les années rapportées (Tableau 1). Si l'on ne compare que les pertes causées par les maladies et les insectes dans les trois principales provinces forestières, on a déjà une meilleure perception des ordres de grandeur des pertes de volumes ligneux en cause pour chacune des deux catégories de ravageurs (Tableau 2). On note qu'en Colombie-Britannique, les pertes par les maladies sont plus élevées que celles causées par les insectes. Les pourridiés représentent une partie importante de ce volume de pertes par les maladies car $91 \%$ de celles-ci sont attribuées aux caries de tiges et de racines.

En Ontario, il y a presque deux fois plus de dommages causés par les maladies que par les insectes. Fait à noter, les évaluations des pertes par les pourridiés en Ontario ont été prises en compte suite aux compilations pour chacune des régions de cette province par Gross et al. (1992). Ces données sont basées sur les travaux de recherche menés au Centre de foresterie des Grands Lacs sur les pourridiés. Environ le tiers des pertes par les maladies sont dues aux caries de tiges et de racines.

Au Québec, les pertes dues aux insectes sont sensiblement équivalentes à celle de l'Ontario et les deux tiers de ces pertes sont attribuées à la tordeuse des bourgeons de l'épinette. Pour ce qui est des pertes par les maladies, on note une certaine aberration car le volume est nettement plus faible qu'en Ontario, et ce, malgré une superficie de terres forestières plus grande au Québec qu'en Ontario. En poussant plus loin notre analyse de ce rapport, on peut enfin comprendre cet écart. En effet, n'ayant pas de données pour les pertes par les pourridiés au Québec, les auteurs les ont estimées à zéro, ce qui est totalement inadmissible (Tableau 3). C'est un secret qui était bien gardé depuis plus d'une décennie. En prenant les données du tableau 17 du rapport de Hall et Moody (1994), on peut estimer les pertes par les pourridiés au Québec en calculant la différence entre le volumes de pertes dues aux carie de tiges et de racines en Ontario moins le volume dû aux caries des tiges au Québec, ce qui donne une approximation du volume de pertes par les pourridiés à $3340000 \mathrm{~m}^{3}$ pour le Québec. Cette évaluation des pertes annuelles bien que non basée sur des relevés réels faits au Québec, nous semble plus près de la réalité et plus plausible qu'un volume nul étant donné les ressemblances des écosystèmes entre les deux provinces. De plus, tous les volumes calculés pour les pertes par les maladies dans les tableaux 1, 2 et 3 doivent être corrigés en conséquence. Ainsi, au tableau 1, on devrait lire 54,5 millions de $\mathrm{m}^{3}$ au lieu de 51,2; au tableau 2, on devrait lire 9,369 millions de $\mathrm{m}^{3}$ au lieu de 6,029; au tableau 3, c'est 5948000 millions de $\mathrm{m}^{3}$ qui devrait apparaître au lieu de 2608000.

\section{Mise en contexte}

Les raisons pour lesquelles on tend à ignorer les pertes causées par les pourridiés sont multiples. D'abord, les pourridiés sont des maladies peu visibles et donc difficiles à inventorier. Les signes de maladies sont souvent fugaces, $n$ 'apparaissant que durant une courte période de l'année. Chez le pourridié-agaric, cette période ne dépasse guère 15 jours. Aussi, le cycle biologique des pourridiés est la plupart du temps très long. Les dommages ne peuvent donc pas être mesurés sur une base annuelle. Lorsque la mortalité causée par ces maladies apparaît, il est souvent déjà trop tard pour intervenir dans le peuplement. Par exemple, les symptômes de la maladie du rond causée par le champignon pathogène Heterobasidion annosum (Fr.) Bref. ne sont apparus que 7 ans après la coupe d'éclaircie dans une plantation de pins rouges (Laflamme et Blais 1995); il est connu que c'est lors de la coupe des arbres que la maladie envahit un site. Dans une autre de nos études sur la carie rouge alvéolaire (Inonotus tomentosus (Fr.:Fr.) Bref.), la mortalité a débuté vers l'âge de 40 ans dans une plantation d'épinettes blanches et la maladie devait y être présente depuis au moins une quinzaine d'années.

Tableau 2. Décroissement annuel par les maladies et les insectes dans trois provinces canadiennes (milliers de mètres cubes) (Hall et Moody 1994)

\begin{tabular}{|c|c|c|c|}
\hline Catégories & Colombie-Britannique & Ontario & Québec \\
\hline $\begin{array}{l}\text { Maladies } \\
\text { Insectes }\end{array}$ & $\begin{array}{l}20477 \\
16635\end{array}$ & $\begin{array}{l}20586 \\
11250\end{array}$ & $\begin{array}{r}6029 \\
10309\end{array}$ \\
\hline $\begin{array}{l}\text { Superficie forestière } \\
\text { (millions ha) }\end{array}$ & 60,6 & 58,0 & 83,9 \\
\hline
\end{tabular}


Tableau 3. Décroissements annuels par les caries de tiges et de racines dans trois provinces canadiennes (milliers de mètres cubes) (Hall et Moody 1994)

\begin{tabular}{llll}
\hline Catégories & Colombie-Britannique & Ontario & Québec \\
\hline Caries & 18657 & 5948 & $2608^{\mathrm{a}}$ \\
\hline
\end{tabular}

${ }^{a}$ Caries de la tige seulement.

En contrepartie, les dommages causés par les insectes défoliateurs sont relativement faciles à inventorier, et ce, sur une base annuelle. C'est sans doute ce qui explique que dans les statistiques publiées annuellement par le Service canadien des forêts, on ne rapporte que les aires forestières dévastées par ces insectes (L'état des forêts au Canada 2003-04, http://www.nrcan-rncan.gc.ca/cfs-scf/national/ what-quoi/sof/latest_f.html). Le fait de concentrer les efforts sur des ravageurs simples à quantifier sur une base annuelle ne présente pas objectivement l'état de santé de nos forêts. On ignore ainsi des ravageurs encore plus importants en termes de pertes ligneuses et au Québec en particulier, les pourridiés en font partie. De plus, cette façon de faire tend à réduire nos écosystèmes à un très petit nombre de composantes. Ainsi, des écologistes de la dynamique naturelle des écosystèmes forestiers ne considèrent pas les maladies fongiques, donc encore moins les pourridiés dans leurs études. On assume que la tordeuse des bourgeons de l'épinette est le facteur responsable de la structure du couvert forestier (D'Aoust et al. 2004). On croit aussi qu'en absence de feux comme principal agent perturbateur, le vent et les insectes auraient un rôle prédominant (Pham et al. 2004), bien que les trouées décrites ressemblent étrangement à des ronds de mortalité causés par des pourridiés. De plus, les chablis qu'on assume n'être causés que par le vent sont souvent associés à un fort pourcentage de pourridiés dans un peuplement.

\section{Quelques pourridiés importants au Québec}

1 - Pourridié-agaric

II y moins de 25 ans, on attribuait généralement cette maladie à une espèce de champignon, Armillaria mellea (Vahl:Fr.) P. Kumm.; on reconnaissait déjà à ce moment plusieurs variations morphologiques à ce champignon. Aujourd'hui, on connaît une douzaine d'espèces d'armillaire au Canada. Leur distribution est assez bien connue au pays sauf dans les trois provinces maritimes : Île-du-Prince-Édouard, NouveauBrunswick et Nouvelle-Écosse. Au Québec, les espèces suivantes ont été identifiées (Bérubé 1991) : A. ostoyae (Romagnesi) Herink, A. gemina Bérubé \& Dessureault, $A$. sinapina Bérubé \& Dessureault, $A$. calvescens Bérubé \& Dessureault et $A$. mellea, bien que $A$. ostoyae soit la plus commune. Le pourridiéagaric joue un rôle plus important qu'on veut bien l'admettre dans les cas d'épidémies d'insectes car lors de relevés que j'ai effectués à Terre-Neuve, les arbres morts dans un peuplement défolié par la tordeuse des bourgeons de l'épinette montraient des signes du pourridié-agaric; des résultats semblables ont aussi été publiés ailleurs (Raske et Sutton 1986).
Le pourridié-agaric est récemment devenu populaire lorsqu'il fut classé dans la catégorie des "monstres". Grâce à des techniques de biologie moléculaire, des chercheurs américains ont réussi à identifier un individu d'armillaire qui s'étendait sur 15 ha, pesait $10000 \mathrm{~kg}$ et était âgé de plus de 1500 ans (Smith et al. 1992). Ce champignon venait de rejoindre le club des baleines et des séquoias. Mais ce monstre a vite été détrôné par des armillaires communs encore plus gigantesques qui couvraient respectivement des surfaces de 20, 95, 260 et 965 ha (Ferguson et al. 2003).

Comme le champignon $A$. ostoyae couvre parfois de vastes superficies sans nécessairement causer un fort taux de mortalité, il semble en équilibre avec le milieu et ne s'attaquerait qu'aux arbres affaiblis ou en état de stress. On a aussi souvent remarqué en plantations de conifères que des souches de feuillus servent de substrat à ce champignon qui s'attaque aux jeunes semis de conifères plantés sur ces sites. Les dommages causés par le pourridié-agaric sont très variables, non seulement entre les régions, mais aussi à l'intérieur d'un peuplement forestier (Wargo et Shaw 1985). Le calcul des pertes ligneuses par le pourridié-agaric est compliqué par le fait qu'elles sont souvent associées à d'autres pourridiés, à des scolytes de l'écorce, à des insectes défoliateurs, ou à des agents abiotiques comme le vent.

\section{2 - Carie rouge alvéolaire}

Ce pourridié causé par le champignon I. tomentosus est présent surtout chez les épinettes, à travers tout le Canada, et provoque une carie rouge alvéolaire qui lui donne son nom. En effet, le bois se colore en rouge après l'attaque du champignon; par la suite, la carie avancée montre des alvéoles tapissées de mycélium blanc.

Même si les données sont fragmentaires, cette maladie est très fréquente au Québec d'après les résultats d'une étude sur les épinettes noires (Lavallée 1987). Parmi les trois régions étudiées, les plus affectées étaient respectivement Charlevoix, Baie-Comeau et la Réserve des Laurentides. Dans une autre région où nous avons fait des observations, soit à Harrington au nord de Lachute, cette maladie ravageait une plantation d'épinettes blanches de 50 ans; le champignon avait causé $29 \%$ de mortalité et $56 \%$ des arbres résiduels étaient affectés. Des résultats semblables ont été rapportés en Mauricie (Ouellette 1967). Dans la région de la Baie-des-Chaleurs, un forestier professionnel qui y a travaillé pendant de nombreuses années nous rapportait que les pertes ligneuses dues à cette maladie étaient parmi les plus importantes que I'on connaisse. Dans la forêt nationale de Petawawa, on ne reboise plus d'épinettes car elles ne peuvent survivre à l'attaque de $I$. tomentosus sur ces sites et meurent à l'âge de 40 ans environ. 


\section{3 - Maladie du rond}

Cette maladie est appelée ainsi car le champignon se propage à partir d'un point central, habituellement une souche infectée, en rayonnant vers l'extérieur, tuant les arbres autour de cette souche (Laflamme 1994). Comme le pourridié-agaric, cette maladie a longtemps été attribuée à une seule espèce de champignon Heterobasidion annosum. Aujourd'hui, en Europe, on reconnaît au moins trois espèces (Niemelä et Korhonnen 1998). La taxonomie du champignon est sous étude en Amérique du Nord et autant d'espèces nouvelles pourraient être décrites. La maladie du rond est connue depuis sa première description par Hartig (1874), mais le mode d'introduction de la maladie par l'infection des souches fraîchement coupées n'a été identifié qu'un demisiècle plus tard (Rishbeth 1951). Cette découverte a permis d'appliquer des moyens de lutte préventifs développés d'abord en Grande-Bretagne. En effet, comme les souches n'existent pas naturellement, on crée une nouvelle niche écologique en coupant des arbres. Les souches de pin rouge de l'Est canadien sont, entre autres, un milieu propice au développement de cette maladie.

Divers produits ont été expérimentés afin de prévenir la colonisation des souches de conifères par $H$. annosum. Nous pensons que I'utilisation d'un champignon saprophyte, Phlebiopsis gigantea (Fr.: Fr.) Jülich, pourrait figurer avantageusement parmi les moyens de prévention de la maladie. En inoculant les souches fraîchement coupées avec ce champignon, nous empêchons sa colonisation par $H$. annosum. De plus, $P$. gigantea va décomposer les souches et prévenir la prolifération du champignon pathogène qui serait déjà présent sur ce site. Toutefois, à cause des règlements très stricts régissant l'utilisation de produits biologiques, il sera très difficile d'homologuer un tel produit au Canada car le marché est restreint et de nombreux tests coûteux sont exigés malgré plus de 50 ans d'utilisation de $P$. gigantea en Grande-Bretagne, sans qu'on ait rapporté d'effets négatifs pour les utilisateurs ou l'environnement. D'autre part, les risques que la maladie se propage dans l'aire de distribution du pin rouge sont de $100 \%$. On peut donc se demander ce que vise à protéger cette réglementation.

\section{Impact croissant des pourridiés sur nos forêts}

Même si au Québec l'impact des pourridiés était un secret bien gardé, on sait maintenant qu'ils causent des pertes ligneuses parmi les plus importantes en forêt, et ce, tant par la mortalité des tiges que par la diminution de croissance des arbres. Par contre, à part la maladie du rond, on connaît peu le développement de ces maladies; sans cette connaissance sur l'épidémiologie de ces champignons pathogènes, on ne peut prétendre développer des moyens efficaces d'intervention, ce qui laisse les forestiers sans possibilité d'aménagement durable des peuplements.

Les pertes ligneuses en forêt vont fort probablement augmenter avec les coupes, le reboisement, les interventions sylvicoles comme les éclaircies et les préparations de terrain. Les observations de Warren (2004) illustrent bien cette appréhension. En effet, son relevé sur les tiges résiduelles de sapin baumier après une éclaircie précommerciale montre des impacts négatifs significatifs dus à la diminution de croissance, à la mortalité et aux chablis; de même, un taux plus élevé de pourridiés est observé. En fait, c'est le résultat inverse que vise l'éclaircie précommerciale dont les objectifs sont d'augmenter le diamètre des arbres résiduels et le volume marchand tout en réduisant l'âge de la rotation. Warren a de plus observé que des champignons jusque là non impliqués dans les maladies de racines ont été fréquemment identifiés comme responsables de pourridiés.

Le développement des méthodes pour les relevés des pourridiés va aider l'optimisation de la récolte de bois de qualité. En effet, des pertes ligneuses attribuées à d'autres causes (chablis, tordeuse des bourgeons de l'épinette) seront mieux diagnostiquées. Les travaux menés au Manitoba (Knowles 2003) montrent bien l'impact de ces connaissances sur la décision des aménagistes forestiers. Ainsi, à partir de relevés détaillés des pourridiés dans une pessière, l'auteur a estimé à $14 \%$ les pertes en volume en l'an 2000 et la projection pour 2016 atteignait déjà $31 \%$ de pertes en volume. Les aménagistes forestiers ont donc changé leur décision pour une récolte hâtive du peuplement.

\section{CONCLUSION}

Ces propos ne se veulent pas alarmistes. L'intention est tout simplement de porter de nouveau à votre attention le problème réel et majeur des pourridiés dans nos forêts puisque ces ravageurs semblent avoir été oubliés en protection des forêts et ne sont même pas considérés lors d'études de nos écosystèmes forestiers. Ils ne figurent pas non plus sur la liste des sujets de recherche à "la saveur du jour». Et pourtant, des recherches sur l'épidémiologie de ces ravageurs devraient être une priorité car, sans ces connaissances, les moyens de lutte contre ces ravageurs ne pourront pas être développés.

\section{RÉFÉRENCES}

Abgrall, J.F. et A. Soutrenon. 1991. La forêt et ses ennemis. $3^{e}$ éd. CEMAGREF, Grenoble, France. 399 pp.

Basham, J.T. 1991. Stem decay in living trees in Ontario's forests: a user's compendium and guide. Info. Rep. O-X-408. Forestry Canada, Ontario Region, Great Lakes Forestry Centre. 64 pp.

Bérubé, J. 1991. Caractérisation morphologique protéique et enzymatique du genre Armillaria. Thèse de doctorat. Faculté de foresterie et de géomatique, Université Laval, Québec. 95 pp.

Boulet, B. 2003. Les champignons des arbres de l'Est de l'Amérique du Nord. Les Publications du Québec, Québec, Canada. 727 pp.

D'Aoust, V., D. Kneeshaw et Y. Bergeron. 2004. Characterization of canopy openness before and after a spruce budworm outbreak in the southern boreal forest. Can. J. For. Res. 34 : 339-352.

Ferguson, B.A., T.A. Dreisbach, C.G. Parks, G.M. Filip et C.L. Schmitt. 2003. Coarse-scale population structure of pathogenic Armillaria species in a mixed-conifer forest in the Blue Mountains of northeast Oregon. Can. J. For. Res. $33: 612-623$. 
Gross, H.L., D.B. Roden, D.B. Churcher, G.M. Howse et D. Getridge. 1992. Pest-caused depletions to the forest resource of Ontario, 1982-1987. Forestry Canada / Ontario Ministry of Natural Resources, Joint Report 17. $16 \mathrm{pp}$.

Hall, J.P. et B. Moody. 1994. Décroissement causé par les insectes et les maladies des arbres au Canada de 1982 à 1987. Relevé des insectes et des maladies. Rapport d'information ST-X-8. Service canadien des forêts, Ressources naturelles Canada, Ottawa. 15 pp.

Hartig, R. 1874. Wichtige Krankheiten der Waldbäume. Beiträge zur Mycologie und Phytopathologie für Botaniker und Forstmänner. Berlin. J. Springer.

Knowles, K. 2003. Shade lake root disease survey. Pages 439-440 in G. Laflamme, J.A. Bérubé et G. Bussières (éds.), Root and butt rots of forest trees: 10th International conference on root and butt rot. Proc. of the IUFRO Working Party 7.02.01; Québec, Canada, Sept. 16-22, 2001. Rapport d'information LAU-X-126.

Laflamme, G. 1994. La maladie du rond causée par Heterobasidion annosum. Feuillet d'information CFL 27. Centre de foresterie des Laurentides, Service canadien des forêts, Ressources naturelles Canada, Québec. 12 pp.

Laflamme, G. et Blais, R. 1995. Détection du Heterobasidion annosum au Québec. Phytoprotection 76 : 39-43.

Laflamme, G. et M. Lortie. 1972. Relation entre la carie du bois, les défauts extérieurs et différents clones du Populus tremuloides Michx. Nat. Can. 99 : 449-456.

Laflamme, G., J. Meades et G. Eagen. 1977. Wood defect and density studies of living trees: I - Field Guide. Environ. Can., For. Serv. Newfoundland. Forest Research Centre. Info. Rep. N-X-148. St. John's, Nfld. 38 pp.

Lavallée, A. 1986. Les caries du sapin baumier dans le centre et le nord-est du Québec. Rapport d'information LAU-X-70. Centre de foresterie des Laurentides, Service canadien des forêts, Québec, Canada. 21 pp.

Lavallée, A. 1987. Les caries de l'épinette noire dans la forêt boréale au Québec. Rapport d'information LAU-X-76. Centre de foresterie des Laurentides, Service canadien des forêts, Québec, Canada. 16 pp.
Lindsey, J.P. et R.L. Gilbertson. 1978. Basidiomycetes that decay aspen in North America. Bibliotheca Mycologica 63. Ed. J. Camer, Vaduz, Germany. 406 pp.

Lortie, M. 1968. Quelques défauts indicateurs de carie chez le sapin baumier. Bull. Fonds Rech. Forest., Université Laval, No. 11. 38 pp.

Niemelä, T. et K. Korhonen. 1998. Taxonomy of the genus Heterobasidion. Pages 27-33 in S. Woodward, J. Stenlid, R. Karjalainen et A. Hüttemann (éds.), Heterobasidion annosum: biology, ecology, impact and control. CAB International, Oxon, UK.

Ouellette, G.B. 1967. Quelques maladies importantes des plantations de conifères dans le Québec. Phytoprotection $48: 86-91$

Ouellette, G.B. et G. Bard. 1961. Notes on the occurrence of Armillaria root rot. Bi-Month. Progr. Rep. $17:$ 1-2.

Pham, A.T., L. De Grandpré, S. Gauthier et Y. Bergeron. 2004. Gap dynamics and replacement patterns in gaps of the northeastern boreal forest of Quebec. Can. J. For. Res. 34 : 353-364.

Raske, A.G. et W.J. Sutton. 1986. Decline and mortality of black spruce caused by spruce budworm defoliation and secondary organisms. Info. Rep. N-X-236. Newfoundland Forestry Centre, Canadian Forest Service, St. John's, Nfld., Canada. 29 pp.

Rishbeth, J. 1951. Observations on the biology of Fomes annosus with particular reference to East Anglia pine plantations II. Spore production, stump infection, and saprophytic activity in stumps. Ann. Bot. $15: 1-21$.

Smith, M.L., J.N. Bruhn et J.B. Anderson. 1992. The fungus Armillaria bulbosa is among the largest and oldest living organisms. Nature $356: 428-431$.

Wargo, P.M. et C.G. Shaw. 1985. Armillaria root rot: the puzzle is being solved. Plant Dis. $69: 826-832$.

Warren, G. 2004. Root and butt rots in semi-mature, pre-commercially thinned stands of balsam fir in Newfoundland. Forest pest management forum. Nov. 2004. Ottawa. http://forum.glfc.forestry.ca. 